Original Research

\title{
Masseter Muscle Fatigue Induced by Experimental Sustained Light Clenching
}

\author{
Ishii Harunaka, DDS, PhD, Piquero Katiuska, DDS, PhD and Sakurai Kaoru, DDS, PhD \\ Department of Complete Denture Prosthodontics, Tokyo Dental College
}

\section{Clinical significance}

Most patients with diurnal bruxism are not aware of this habit. In this study, a straight regression line representing the characteristics of masseter muscle fatigue was plotted when subjects were asked to perform experimental light clenching. This finding is very important, since it can be used in dental practice as a diagnostic tool to assess masseter muscle fatigue at an early stage in those patients who are not aware they have diurnal bruxism, especially the continuous, light clenching type.

\begin{abstract}
Purpose: The aim of this study was to clarify the characteristics of masseter muscle fatigue induced by experimental sustained light clenching with rest intervals between clenching events.

Materials and Methods: The subjects consisted of 18 healthy young adults. For each recorded task, the subjects were asked to perform experimental tooth clenching 3 times, with a duration of $60 \mathrm{~s}$ for each event and an interval ( $30 \mathrm{~s}$ or $60 \mathrm{~s})$ between successive clenching events. The experimental clenching forces selected were $10 \%$ and $20 \%$ of maximum voluntary clenching (MVC). The electromyographic activity during the experimental procedure was measured. The mean power frequency (MPF) of muscle activity was used as an indicator of muscle fatigue. Changes to lower frequencies of the MPF were then analyzed.

Results : Despite the light clenching forces used (10\% and $20 \%$ of MVC), masseter muscle fatigue occurred. When the subjects performed experimental clenching at $10 \%$ of MVC with intervals of $60 \mathrm{~s}$ between clenching events, the MPF changes were gradually larger depending on the events. The regression line had the best fit to data when the subjects were asked to perform experimental clenching under the conditions mentioned above.
\end{abstract}

Conclusion: It was possible to obtain a regression line that represented the relationship between clenching events and masseter muscle fatigue.

\section{Key words}

masseter muscle, muscle fatigue, clenching, EMG (Electromyography)

Corresponding author: Ishii Harunaka

1-2-2, Masago, Mihama-Ku, Chiba 261-8502, Japan

Tel : +81-43-270-3933, Fax : +81-43-270-3935

E-mail : hishii@tdc.ac.jp

Received on February 1, 2005/Accepted on June 8, 2005 


\section{INTRODUCTION}

Bruxism has been mentioned as one of the physical means of releasing tension from emotional stress, and its effects not only on the stomatognathic system but also on the entire body have received considerable attention ${ }^{1-3)}$. In recent years, investigations examining the diagnostic criteria of bruxism ${ }^{4}$, as well as the influence of bruxism on the mucosa underlying dentures ${ }^{5}$, have been performed. Since stress and anxiety have been discussed as playing major roles in causing bruxism, and considering that people are frequently subjected to high levels of stress, the understanding of bruxism behavior is extremely important.

During regular dental visits, it is not uncommon for the dentist to encounter patients who complain of masticatory muscle fatigue and/or pain, especially localized at the masseter muscle. Some of these patients are aware of their habit (especially in the case of clenching), while others are not. Ikeda et al. ${ }^{6}$ established diagnostic criteria for the detection of sleep-associated bruxism in patients. Piquero and Sakurai ${ }^{7)}$ applied these criteria in order to determine a diagnostic method for detecting diurnal bruxism, and they concluded that there were different individual patterns of diurnal bruxism, including a clenching type characterized by low-voltage amplitude levels of long duration.

Other studies have investigated the jaw muscle fatigue and muscle pain induced by experimental clenching or mastication ${ }^{8-11)}$. These studies have led to a better understanding of the masseter muscle physiology. However, most of these studies have used clenching forces as strong as $50 \%-80 \%$ of maximal voluntary clenching for 3-5 minutes, whereas other studies have used light force for the experimental clenching, but sustained for as long as 60 minutes. In previous studies, it was demonstrated that it is common to observe the presence of intervals during which there is no muscular activity between the sustained light clenching events ${ }^{7}$. It is not known yet if this type of clenching leads to jaw muscle fatigue or not. Even if muscle fatigue occurs as a consequence of this type of repeated sustained light clenching, the characteristics of the muscle fatigue are not well understood. It is believed that individual differences in the level of muscle fatigue depend on the severity of bruxism. We hypothesized that if it were possible to find a certain tendency in masseter muscle fatigue as a consequence of repeated experimental light clenching performed by healthy subjects, then we would be able to obtain an indicator of the clinical condition of a healthy masseter muscle. Therefore, the aim of this study was to clarify the characteristics of masseter muscle fatigue induced by experimental sustained light clenching with intervals of rest between the clenching events.

\section{MATERIALS AND METHODS}

\section{Subjects}

The subjects consisted of 18 healthy young adults (9 males and 9 females; mean age 26士2) having normal dentition. No signs of diurnal bruxism as well as abnormalities of the stomatognathic system were found. The results obtained from a clinical examination based on a questionnaire and palpation of muscles revealed that there was no fatigue sensation and/or pain in the masseter and other masticatory muscles. In addition, to assess the presence of diurnal bruxism, the criteria for the detection of diurnal bruxism $^{7)}$ were applied after recording the masseter muscle activity of each subject during 10 minutes of silent reading. None of them showed diurnal bruxism. All subjects were fully informed about the experimental procedures, and they 
gave their informed consent to participate in the study.

\section{Recording System and Location}

An electromyographic (EMG) recording device Muscle Tester ME $3000 \mathrm{p}$ (Mega Electronics, Finland), with a built-in 12 bit A/D converter, was used to record the myoelectrical muscle activity. Bipolar surface electrodes (Blue Sensor $\mathrm{P}-00-\mathrm{S}$, Medicotest, Denmark) were placed in the direction of the muscle fibers over the main bulk of the right masseter muscle, as determined by palpation ${ }^{12)}$, with an interelectrode distance of $20 \mathrm{~mm}$. The sampling frequency selected was $1000 \mathrm{~Hz}$. Before placing the electrodes, the skin was thoroughly cleansed using a skin cleansing gel (Nihon Kohden, Japan) and ethanol-soaked gauze. The skin impedance between the electrodes was lower than $8 \mathrm{k} \Omega$. The measured myoelectrical activity was downloaded into a personal computer through an optical fiber for further analyses.

\section{Experimental Clenching}

Each subject was asked to clench their masseter muscle at $10 \%$ and $20 \%$ of their maximum voluntary clenching (MVC). The subjects were able to control their EMG levels through visual feedback by looking at the computer displaying the voltage amplitudes.

\section{Recording Procedure}

The same recording procedure was carried out for both clenching forces (10\% and $20 \%$ of $\mathrm{MCV})$. For each recorded task, the subjects were asked to perform experimental clenching 3 times (called "event 1, event 2 and event 3 ") for $60 \mathrm{~s}$ per event with intervals of $30 \mathrm{~s}$ and $60 \mathrm{~s}$ between each successive clenching event. The tasks to be recorded were as follows:

1. Task 1 : clenching at $10 \%$ of MVC $; 30^{-} \mathrm{s}$ intervals.
2. Task 2 : clenching at $10 \%$ of MVC $; 60^{-} \mathrm{s}$ intervals.

3. Task 3 : clenching at $20 \%$ of MVC $; 30-\mathrm{s}$ intervals.

4. Task 4 : clenching at $20 \%$ of MVC $; 60^{-}$s intervals.

The experimental design is shown in Fig. 1. Three trials of these recorded tasks were performed on different days.

\section{Data Processing and Analysis}

The measured values were analyzed using an exclusive software program (Mega Win Software Version 1.1, Mega Electronics, Finland). For each event, a Fast Fourier Transform (FFT) algorithm was used to calculate the mean power frequency (MPF) of the muscle activity. Two values were measured : a) the shift of the mean power frequency value (change in MPF) to lower frequencies as an indicator of the muscle fatigue for each event; and b) the MPF value at the beginning of each event (initial MPF) in order to record the fatigue recovery at the beginning of each consecutive event following the interval. The initial MPF was calculated as the MPF value during the first $0.16 \mathrm{~s}$ (using a commercial analysis program) of each event. Comparisons among the change in MPF and the initial MPF for each event were carried out by means of repeated measures ANOVA. Subsequently, a paired $t$-test was applied using a probability level that was adjusted by Bonferroni's inequality.

To examine the correlation between the two variables (in this case, the change in MPF and the experimental clenching events), a linear regression analysis was employed. In all tests, statistical significance was accepted at a probability level of $\mathrm{p}<0.05$ by using SPSS $11.0 \mathrm{~J}$ (SPSS, USA). 
$10 \%$ of MVC

Experimental Clenching Interval

Task
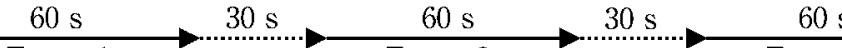

Task 2

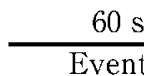

$60 \mathrm{~s} \quad 60 \mathrm{~s}$

Event 2

Event 3

$20 \%$ of MVC

Experimental Clenching Interval

Task 3

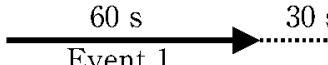

s $60 \mathrm{~s}$

$60 \mathrm{~s} \quad 30 \mathrm{~s}$

$60 \mathrm{~s} \longrightarrow 60 \mathrm{~s}$

$60 \mathrm{~s}$

Event 3

Task 4

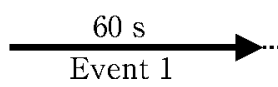

$60 \mathrm{~s}$
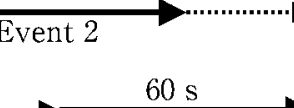

$60 \mathrm{~s}$

Event 3

$60 \mathrm{~s}$

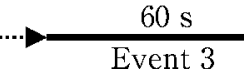

Measured values

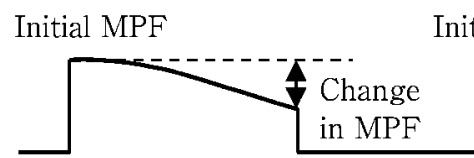

Event 1
Initial MPF

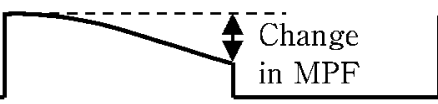

Event 2
Initial MPF

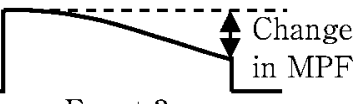

Event 3

Fig. 1 Experimental design

Table 1 Results of repeated measures ANOVA for change in MPF

\begin{tabular}{lcrrr}
\hline \hline & $\begin{array}{c}\text { Degrees of } \\
\text { freedom }\end{array}$ & Mean square & F-value & p-value \\
\hline Task 1 & 2 & 208.963 & 65.740 & $0.000^{*}$ \\
Task 2 & 2 & 460.074 & 223.938 & $0.000^{*}$ \\
Task 3 & 2 & 300.167 & 29.639 & $0.000^{*}$ \\
Task 4 & 2 & 23.130 & 3.443 & $0.043^{*}$ \\
\hline & & \multicolumn{3}{c}{$* \mathrm{p}<0.05$}
\end{tabular}

\section{Experimental Ethics}

The protocol was approved by the Ethics Committee of Tokyo Dental College (027). All the experiments were done in accordance with the Edinburgh Revision of the Helsinki Declaration.

\section{RESULTS}

The results of repeated measures ANOVA for the changes in MPF are shown in Table 1. The means and standard deviations for the change in MPF and initial MPF for each event for all the tasks are shown in Tables 2 and 3, respectively.
Table 2 Results of change in MPF at each event

\begin{tabular}{ccccc}
\hline \hline & & Event 1 & Event 2 & Event 3 \\
\hline \multirow{2}{*}{ Task 1 } & Mean & -7.4 & -13.9 & -12.5 \\
& SD & $(1.8)$ & $(3.5)$ & $(4.0)$ \\
\hline \multirow{2}{*}{ Task 2 } & Mean & -7.1 & -12.4 & -17.3 \\
& SD & $(1.8)$ & $(3.3)$ & $(3.2)$ \\
\hline \multirow{2}{*}{ Task 3 } & Mean & -21.6 & -17.7 & -13.4 \\
& SD & $(3.9)$ & $(4.3)$ & $(5.3)$ \\
\hline \multirow{2}{*}{ Task 4 } & Mean & -20.1 & -20.9 & -18.7 \\
& SD & $(3.3)$ & $(5.8)$ & $(4.5)$ \\
\hline & & & & unit : Hz
\end{tabular}

Table 3 Results of initial MPF at each event

\begin{tabular}{ccccc}
\hline \hline & & Event 1 & Event 2 & Event 3 \\
\hline \multirow{2}{*}{ Task 1 } & Mean & 209.0 & 206.5 & 200.7 \\
& SD & $(24.2)$ & $(24.9)$ & $(25.3)$ \\
\hline \multirow{2}{*}{ Task 2 } & Mean & 207.3 & 206.6 & 206.5 \\
& SD & $(23.6)$ & $(22.6)$ & $(22.3)$ \\
\hline \multirow{2}{*}{ Task 3 } & Mean & 211.3 & 202.7 & 193.3 \\
& SD & $(26.6)$ & $(28.5)$ & $(27.3)$ \\
\hline \multirow{2}{*}{ Task 4 } & Mean & 211.1 & 205.2 & 199.5 \\
& SD & $(23.8)$ & $(24.7)$ & $(24.0)$ \\
\hline & & & & unit: $\mathrm{Hz}$
\end{tabular}

1. Task 1 : clenching events at $10 \%$ of MVC ; 30-s intervals

For the change in MPF, there was a signifi- 


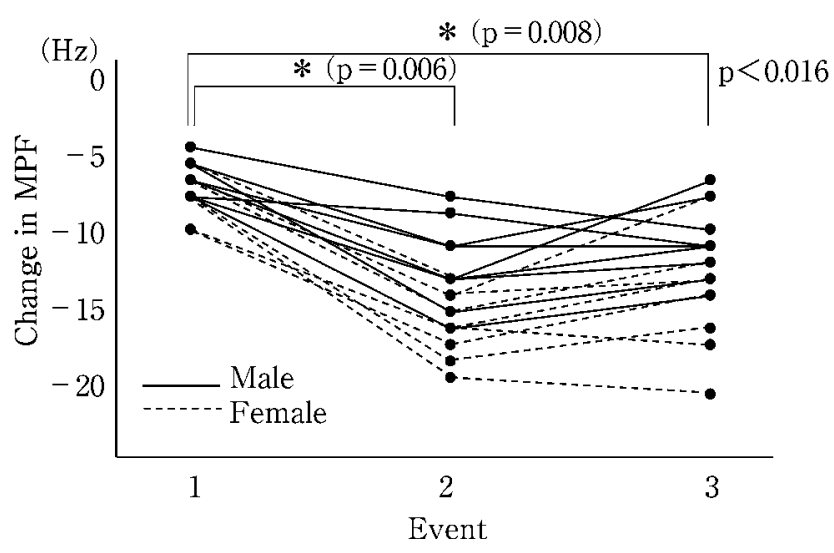

Fig. 2 Changes in MPF according to event (Task 1: clenching events at $10 \%$ of MVC ; $30-$ s intervals)

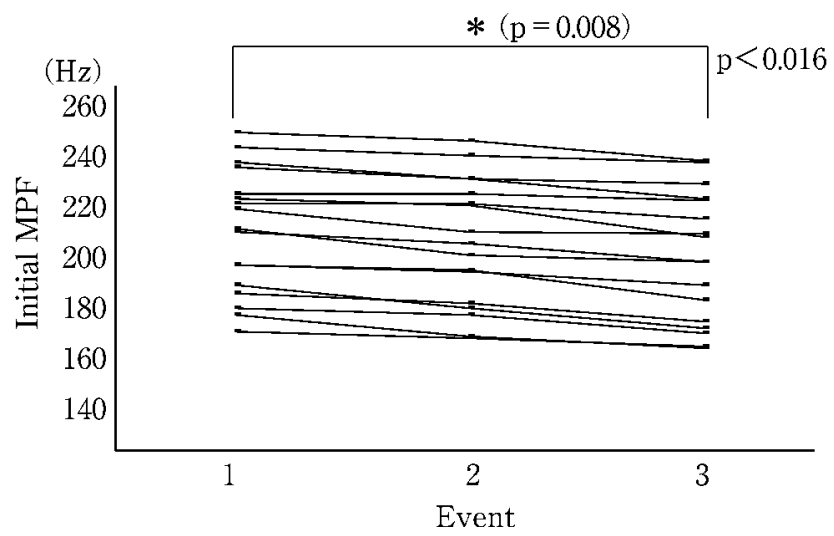

Fig. 3 Initial MPF at each event (Task 1 : clenching events at $10 \%$ of MVC $; 30-$ s intervals)

cant difference between events 2 and 3 compared to event 1 (Fig. 2). For the initial MPF, it was found that there was a significant difference in event 3 compared to event 1 . However, there were no significant differences in the initial MPF between events 1 and 2, and events 2 and 3 (Fig. 3).

2. Task 2 : clenching events at $10 \%$ of MVC ; 60-s intervals

A comparison of the change in MPF among the events showed that there were significant differences between events 2 and 1 , between events 3 and 2, and also between events 1 and 3 (Fig. 4). Observation of the initial MPF values according to the event revealed that there were no significant differences among the events (Fig. $5)$.

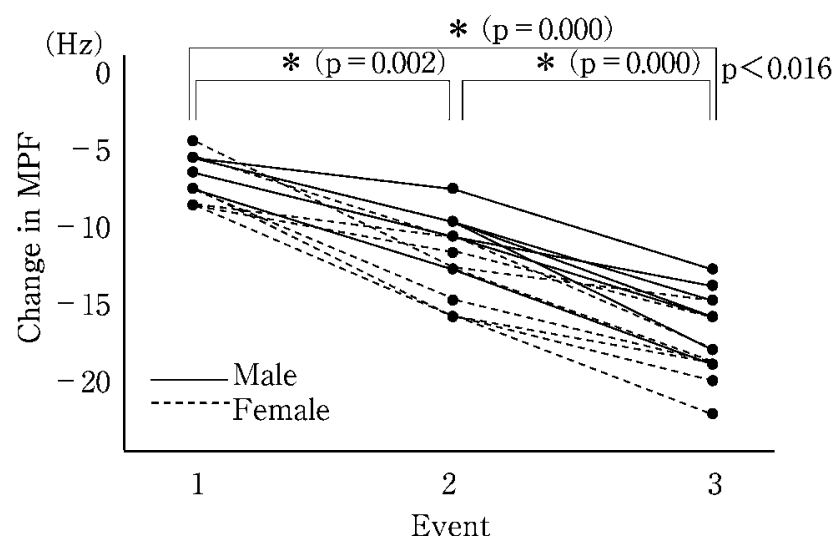

Fig. 4 Changes in MPF according to event (Task 2: clenching events at $10 \%$ of MVC ; $60-$ s intervals)

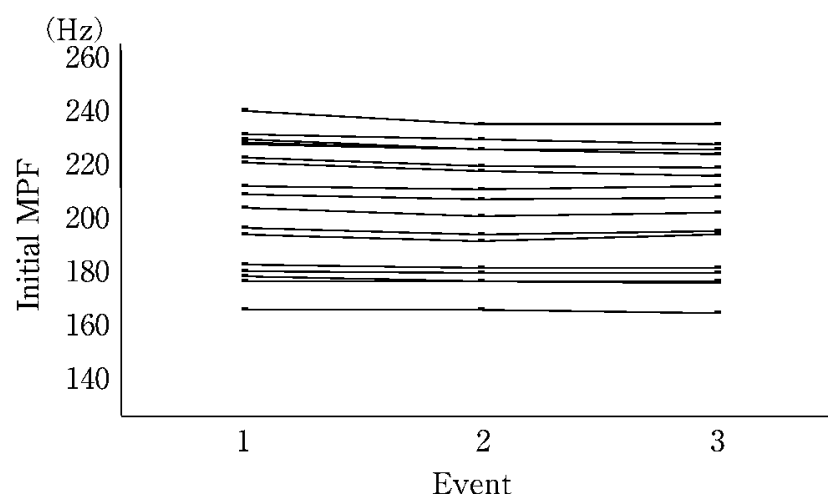

Fig. 5 Initial MPF at each event (Task 2: clenching events at $10 \%$ of MVC ; $60-$ s intervals)

3. Task 3 : clenching events at $20 \%$ of MVC ; 30-s intervals

With regard to the changes in MPF, there was a significant difference between events 1 and 2. The same result was found when comparing the changes in MPF for events 2 and 3 and also events 1 and 3 (Fig. 6). The change in MPF was gradually smaller from events 1 to 3 . It was found that there was a significant difference in events 2 and 3 for the initial MPF compared to event 1 . Also, there was a significant difference in event 3 compared to event 2 (Fig. 7).

4. Task 4 : clenching events at $20 \%$ of MVC ; 60-s intervals

There was a significant difference in event 2 for the changes in MPF compared to event 3 (Fig. 8). With regard to the initial MPF, there 


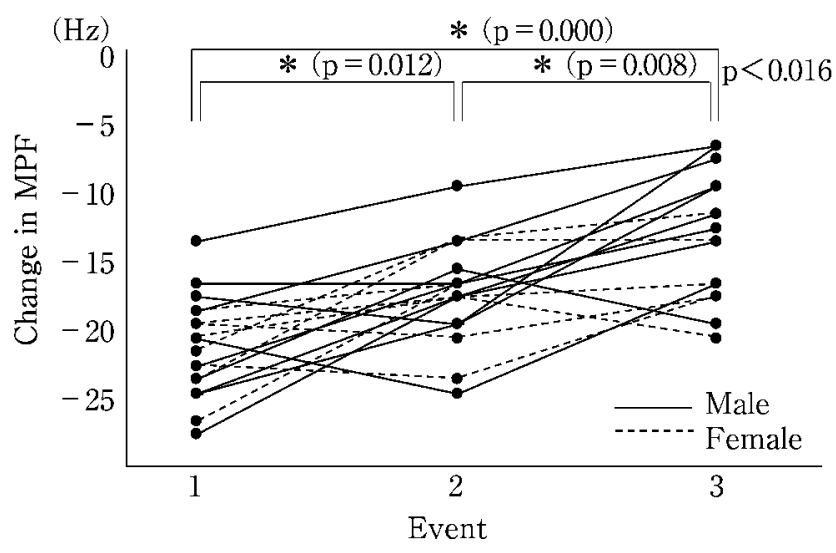

Fig. 6 Changes in MPF according to event (Task 3 : clenching events at $20 \%$ of MVC ; 30 -s intervals)

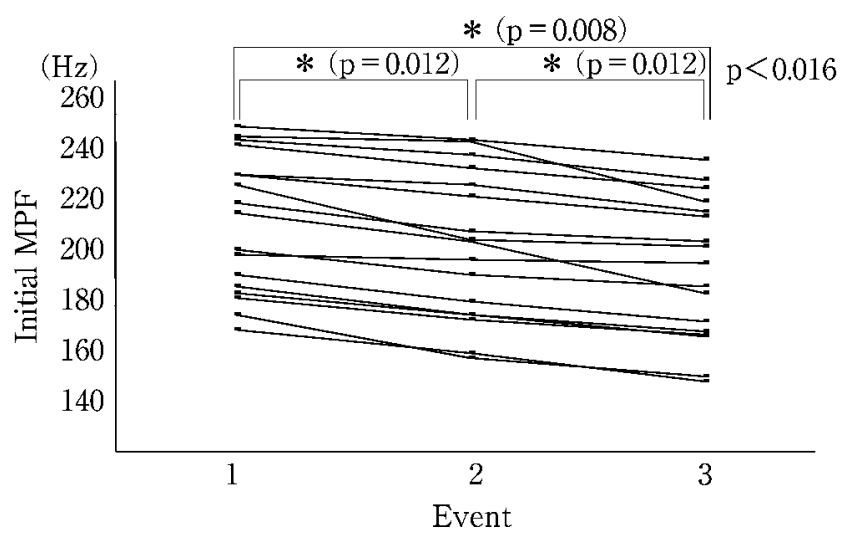

Fig. 7 Initial MPF at each event (Task 3 : clenching events at $20 \%$ of MVC ; $30-$ s intervals)

was a significant difference between events 1 and event 2, and also events 1 and 3 (Fig. 9).

The same interval between events 1 and 2 as between events 2 and 3 is shown in Fig. 2-9. To examine the correlation between the changes in MPF and the experimental clenching, linear regression analyses were applied. Through the calculation of the coefficient of correlation for the regression equation, the goodness of fit was determined for each task. The following regression equations were obtained:

Task $1: \mathrm{Y}=-2.537 \mathrm{X}_{1}-2.556 \mathrm{X}_{2}-4.889$

(Adj- $\mathrm{R}^{2}=0.322 ; \mathrm{p}=0.000$, standard error of estimates $=3.496$ )

Task 2: $\mathrm{Y}=-5.065 \mathrm{X}_{1}-2.556 \mathrm{X}_{2}-0.858$ $\left(\right.$ Adj- $R^{2}=0.749 ; p=0.000$, standard error of estimates $=2.511)$

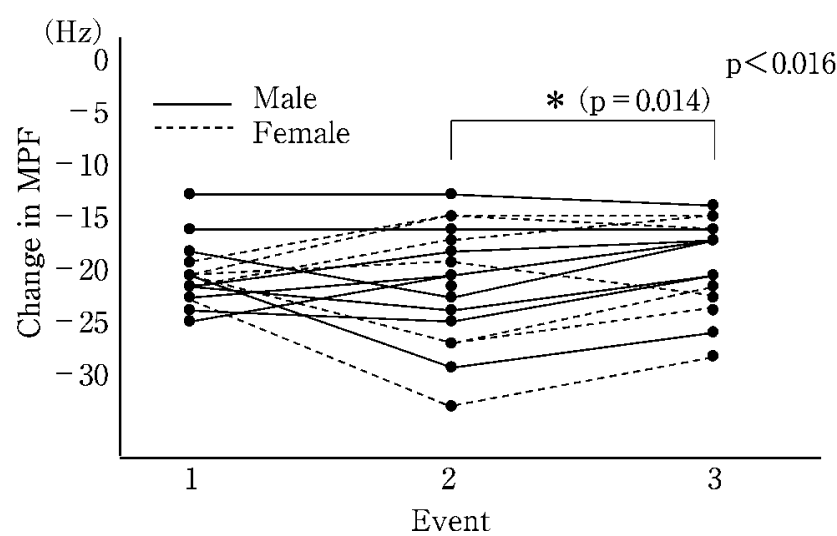

Fig. 8 Changes in MPF according to event (Task 4: clenching events at $20 \%$ of MVC ; 60 -s intervals)

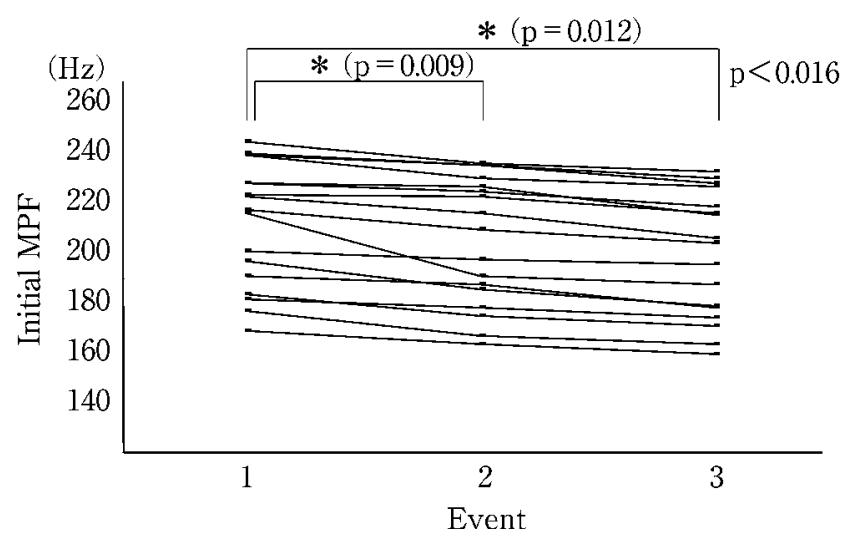

Fig. 9 Initial $\mathrm{MPF}$ at each event (Task 4: clenching events at $20 \%$ of MVC ; $60-$ s intervals)

Task 3: $\mathrm{Y}=4.065 \mathrm{X}_{1}-2.432 \mathrm{X}_{2}-24.488$

$\left(\operatorname{Adj}-R^{2}=0.391 ; p=0.000\right.$, standard error of estimates $=4.358)$

Task $4: \mathrm{Y}=0.704 \mathrm{X}_{1}-2.012 \mathrm{X}_{2}-20.333$

$\left(\operatorname{Adj}-\mathrm{R}^{2}=0.049 ; \mathrm{p}=0.009\right.$, standard error of estimates $=4.581$ )

where $\mathrm{Y}=$ the change in MPF, $\mathrm{X}_{1}=$ the event $\left(\mathrm{X}_{1}\right.$ is 1,2 or 3 ), and $\mathrm{X}_{2}=$ the gender (male : 0 , female $: 1) . \quad A d j-R^{2}=$ the coefficient of determination adjusted for the degree of freedom.

The regression equation fit well for the data obtained from Task 2. In contrast, the regression equation did not fit the data obtained from the other three tasks. 


\section{DISCUSSION}

Christensen reported that the subjective fatigue in the masseter muscle occurred $21 \mathrm{~s}$ after the onset of muscle hyperactivity, and severe muscle pain appeared at about $82 \mathrm{~s}^{8)}$. Based on these results, the experimental clenching duration in the present study was set at $60 \mathrm{~s}$ per event in order to avoid psychological and physiological stressing of the subjects.

To maintain the experimental clenching force at the same level during the experimental procedure, the subjects learned to control their muscle activity level through visual feedback. Since the clenching time selected for the experimental clenching in this study was relatively long $(60 \mathrm{~s})$, it was predicted that an increase in amplitude of the EMG signal would occur. Therefore, an occlusal force measurement device was used prior to the actual experiment to control the experimental clenching force. As a result, it was found that the clenching events performed at $30 \%$ of MVC led to a significant increase in amplitude, while such increases in the amplitude were not recognized for clenching events performed at $10 \%$ and $20 \%$ of MVC. Moreover, it was considered that the presence of a device measuring the occlusal force between the maxillary and mandibular dental arches would lead to a clenching condition much different from physiological clenching. Thus, taking these two aspects into consideration, visual feedback was selected as the method for monitoring the muscle activity.

Two different time intervals were used in this study $(30 \mathrm{~s}$ and $60 \mathrm{~s})$. Hori reported that after termination of a sustained contraction, even as strong as $80 \%$ of $\mathrm{MVC}$, the muscle recovered from fatigue within 3 minutes ${ }^{13)}$. Based on this report and also taking into consideration that the cumulative fatigue, as well as the physiological muscle fatigue sensation, might produce a drop in the concentration necessary to perform the experimental tasks, the above-mentioned interval times were selected.

Until now, studies on muscle fatigue have repeated analyses of the frequency components of the EMG signals ${ }^{13-16)}$. The parameters used to measure the shift toward the lower frequencies in the EMG frequency power spectrum are the Mean Power Frequency (MPF), Median Frequency (MF), and the Zero Cross Rate (ZCR). Hori reported that MPF is the best parameter due to its capacity to reflect the muscle fatigue process, as well as its reproducibility ${ }^{17}$. Thus, MPF was used in the present study as an indicator of muscle fatigue. With regard to the recording location, the main bulk of the right masseter muscle was selected, based on a study performed by Naeije and Zorn who reported that there were no significant differences between the MPF value of the right and left masseter and temporal muscles in healthy subjects ${ }^{18}$.

The MPF values for Task 2 showed a complete recovery to initial levels at the beginning of each event since the interval time used was $30 \mathrm{~s}$ longer than for Task 1. However, the change in MPF gradually became larger according to the events. In this regard, there was a complete recovery of the action potential along the muscle fibers at the beginning of each event. Nevertheless, a complete recovery of muscle fatigue following the sustained contraction was not accomplished, which explained why the amount of change in MPF gradually increased. Moreover, the differences between the results observed at each event in Task 3 and Task 4 were recognized as being due to differences in the intervals used in each task. This finding was especially evident for Task 3, where the initial MPF at each event remarkably diminished. Based on this finding, it was considered that the muscle fatigue reached the highest level at this task compared to the other tasks. It is apparent from these results 
that not only the changes in the MPF level but also the observation of the initial MPF level of each consecutive clenching event should be measured in order to clarify the characteristics of the masseter muscle fatigue.

In the present study, it was possible to assess the characteristics of masseter muscle fatigue in healthy subjects by the observation of the MPF changes induced by experimental clenching. With regard to the relationship between the change in MPF and repeated experimental clenching events, regression analyses were performed for each task. The results obtained from the regression analyses seem to indicate that the regression equation only fits the data obtained from Task 2 (clenching event at 10\% of MVC ; $60^{-} \mathrm{s}$ intervals), which had the best fit of the regression line. In other words, the characteristics of masseter muscle fatigue were clearly expressed after the healthy subjects performed experimental clenching under the Task 2 conditions. For the regression analyses, the subjects' gender was used as one variable, and for this reason, the results obtained from females and males were shown separately in the figures on the change in MPF according to clenching event. Although individual differences were found in the amount of change in MPF, the amounts tended to be almost the same among the subjects. Thus, it was assumed that it would be possible to diagnose the healthiness of the masseter muscle activity. In contrast, there was a wide range of results from the other three tasks among the subjects. Consequently, the regression equation did not fit all data from the other three tasks well.

\section{CONCLUSION}

In conclusion, this study was designed to clarify the characteristics of masseter muscle fatigue induced by experimental sustained light clenching with rest intervals between clenching events.

The results found were :

1. Despite the light clenching forces of only $10 \%$ and $20 \%$ of MVC, masseter muscle fatigue occurred.

2. To evaluate muscle fatigue, it is necessary to observe not only the changes in the MPF as a result of experimental clenching but also to determine the initial MPF at each event as an indicator of muscle recovery.

3. The regression equation had the best fit to the data obtained when healthy subjects were asked to perform experimental clenching for $60 \mathrm{~s}$ at $10 \%$ of MVC with $60-\mathrm{s}$ interval times between clenching events. Therefore, it was possible to obtain a regression line that represents the characteristics of masseter muscle fatigue.

Acknowledgement: We are very grateful to all the subjects who participated in this study. Also we would like to express our thanks to Dr. Mutsumi Takagiwa, Associate Professor, Tokyo Dental College, Mathematics Laboratory, for his valuable assistance with the statistical analysis of the results.

\section{REFERENCES}

1) Faulkner KD. Bruxism: a review of the literature. Part I . Aust Dent J 35:266-276, 1990.

2) Faulkner KD. Bruxism : a review of the literature. Part II. Aust Dent J 35 : 355-361, 1990.

3) Pingitore G, Chrobak V, Petrie J. The social and psychologic factor of bruxism. J Prosthet Dent 65 : 443-446, 1991.

4) Takagi I, Sakurai K. Investigation of the factors related to the formation of the buccal mucosa ridging. J Oral Rehabil 30:565-572, 2003.

5) Akazawa H, Sakurai K. Changes of blood flow in the mucosa underlying a mandibular denture following pressure assumed as a result of light clenching. J Oral Rehabil 29:336-340, 2002.

6) Ikeda $T$, Nishigawa K, Kondo $K$ et al. Criteria for the detection of sleep-associated bruxism in humans. J Orofac Pain 10 : 270-281, 1996.

7) Piquero K, Sakurai K. A clinical diagnosis of diurnal 
(non-sleep) bruxism in denture wears. J Oral Rehabil $27:$ 473-482, 2000.

8) Christensen LV. Progressive jaw muscle fatigue of experimental tooth clenching in man. J Oral Rehabil 8 : 413-420, 1981.

9) Lyons MF, Rouse ME, Baxendale RH. Fatigue and EMG changes in the masseter and temporalis muscles during sustained contractions. J Oral Rehabil 20 : 321-331, 1993.

10) Christensen LV, Tran KT, Mohamed SE. Gum chewing and jaw muscle fatigue and pains. J Oral Rehabil 23 : 424-437, 1996.

11) Svensson P, Burgaard A, Schlosser S. Fatigue and pain in human jaw muscles during a sustained, lowintensity clenching task. Arch Oral Biol 46: 773-777, 2001.

12) Christensen LV. Jaw muscle fatigue and pains induced by experimental tooth clenching : a review. J Oral Rehabil 8:27-36, 1981.

13) Hori H. Electromyographic study of masticatory muscle fatigue and recovery under voluntary contraction. Part 2. Comparison between normal sub- jects and craniomandibular disorder patients. J Jpn Prosthodont Soc $33: 294-302,1989$. (in Japanese)

14) Petrofsky JS, Lind AR. Frequency analysis of the surface electromyogram during sustained isometric contractions. Eur J Appl Physiol 43 : 173-182, 1980.

15) Palla S, Ash MM Jr. Power spectral analysis of the surface electromyogram of human jaw muscles during fatigue. Arch Oral Biol 26 : 547-553, 1981.

16) Sakamoto K, Usui T, Hayami A, Ohkoshi K. The wave analyses with fast fourier transform on surface electromyogram and tremor during an acute and an accumulative fatigue. Electromyogr Clin Neurophysiol $22: 207-228,1982$.

17) Hori H. Electromyographic study of masticatory muscle fatigue and recovery under voluntary contraction. Part 1. Comparison of spectrum parameters for the power spectral shift. J Jpn Prosthodont Soc $33: 37-42,1989$. (in Japanese)

18) Naeije M, Zorn H. Changes in the power spectrum of the surface electromyogram of the human masseter muscle due to local fatigue. Arch Oral Biol 26 : 409-412, 1981. 